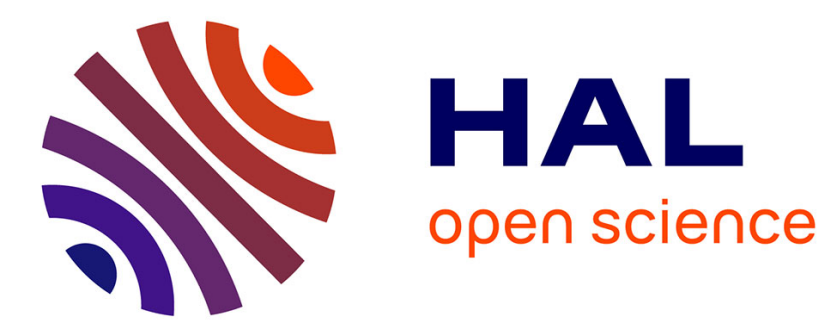

\title{
Plateau borders of smectic liquid crystalline films
}

Torsten Trittel, Ruth Aldred, Ralf Stannarius

\section{To cite this version:}

Torsten Trittel, Ruth Aldred, Ralf Stannarius. Plateau borders of smectic liquid crystalline films. Philosophical Magazine, 2011, pp.1. 10.1080/14786435.2011.558525 . hal-00680174

\section{HAL Id: hal-00680174 https://hal.science/hal-00680174}

Submitted on 18 Mar 2012

HAL is a multi-disciplinary open access archive for the deposit and dissemination of scientific research documents, whether they are published or not. The documents may come from teaching and research institutions in France or abroad, or from public or private research centers.
L'archive ouverte pluridisciplinaire HAL, est destinée au dépôt et à la diffusion de documents scientifiques de niveau recherche, publiés ou non, émanant des établissements d'enseignement et de recherche français ou étrangers, des laboratoires publics ou privés. 


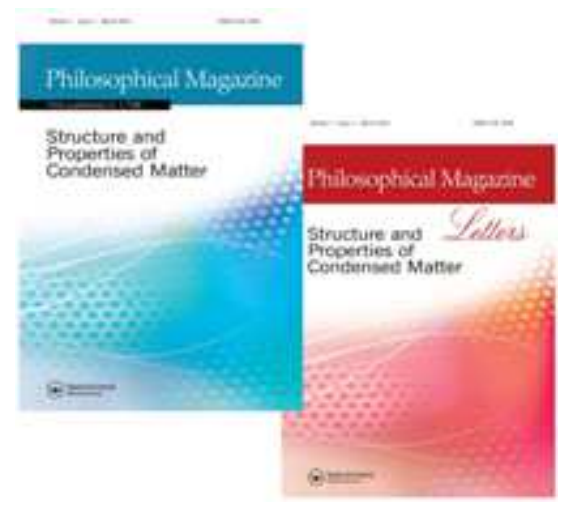

\section{Plateau borders of smectic liquid crystalline films}

\begin{tabular}{|r|l|}
\hline Journal: & Philosophical Magazine \& Philosophical Magazine Letters \\
\hline Manuscript ID: & TPHM-10-Sep-0431.R1 \\
\hline Journal Selection: & Philosophical Magazine \\
\hline Author: & 17-Jan-2011 \\
\hline Complete List of Authors: & $\begin{array}{l}\text { Trittel, Torsten; Otto-von-Guericke Universität, Institut für } \\
\text { Experiemntelle Physik } \\
\text { Aldred, Ruth; University of Exeter, School of Physics } \\
\text { Stannarius, Ralf; Otto-von-Guericke Universität, Institut für } \\
\text { Experimentelle Physik }\end{array}$ \\
\hline Keywords: & foams, liquid crystals, thin films \\
\hline Keywords (user supplied): & \\
\hline & \multicolumn{2}{|l}{} \\
\hline
\end{tabular}

\section{SCHOLARONE" \\ Manuscripts}


Philosophical Magazine Letters

Vol. 00, No. 00, Month 20xx, 1-10

\title{
RESEARCH ARTICLE
}

\section{Plateau borders of smectic liquid crystalline films}

\author{
Torsten Trittel ${ }^{\mathrm{a} *}$, Ruth Aldred ${ }^{\mathrm{b}}$ and Ralf Stannarius ${ }^{\mathrm{a}}$ \\ ${ }^{\mathrm{a}}$ Institut für Experimentelle Physik, Otto-von-Guericke Universität, D-39106 Magdeburg,

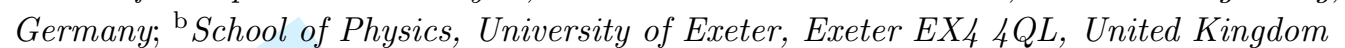

(Received ?? ??? 2010)

\begin{abstract}
We investigate the geometrical properties of Plateau borders in an arrangement of connected smectic A free standing films. The geometry is chosen such that a circular Plateau border surrounds a planar smectic film and connects it with two smectic catenoids. It is demonstrated that similar to soap films, the smectic film geometry can be described by a negative line tension of the circular contact region. Thus, the equilibrium angle between the films depends upon the liquid content in this region, and with increasing liquid content, deviations from Plateau's rule are observed. The experimental results are qualitatively comparable to soap films. A possible origin of slight quantitative differences is discussed.
\end{abstract}

Keywords: foams; Plateau border; Plateau's rules; decoration lemma; thin films; smectic liquid crystals

\section{Introduction}

Foams of different compositions play an important role in everyday's life, including food and beverage industry, technical products for insulation, mechanical damping, cleaning, fire fighting and others. The inner structure of a dry foam is an ensemble of cells formed by thin liquid films. The quasi-polyhedral cells are filled with a gas. Already in the middle of $19^{\text {th }}$ century, Joseph Plateau had expressed the basic scientific concepts describing foam properties, and he established geometrical rules for stable foam structures [1]. Foam dynamics and ageing have attracted the interest of researchers for decades, quantitative relations for many properties of foams have been derived analytically as well as empirically. Numerous experiments have been devoted, and are being devoted, to the study of foam structures and the relation to their mechanical properties [2-4]. Nevertheless, such materials are still fascinating, and even today some of their basic properties are only partially understood. Open questions concern, for example aspects of foam dynamics like drainage and coarsening, $[5,6]$. One interesting aspect that demonstrates the importance of foam research for other fields concerns similarities between the description of cell structures in foams and granular materials $[7,8]$.

The Plateau borders in foam structures, the zones where the liquid films converge, play an important role in the foam dynamics. They contain the main part of the liquid component. When foams are sheared or strained, the reorganization of the cell structure involves viscous flow in the Plateau borders, and dissipation $[9,10]$. But even in absence of external forces, ageing of the foam by gas diffusion through

*Corresponding author. Email: torsten.trittel@student.uni-magdeburg.de 
the liquid films leads to a reorganization of cell ensembles and the motion of Plateau borders.

Mathematical models of foam structures often reduce the cells to quasipolyhedral objects separated by infinitely thin films, which meet in lines that form the skeleton of the foam. This is reflected in the abstract 'dry' foam model, for which simple geometrical rules describe the equilibrium angles between films. For example, the equilibrium angles of three films that meet in a contact line have to be $120^{\circ}$ (Plateau's rule). Only then, forces related to film tensions compensate each other. If the liquid content of the foam is not negligible, in 'real' foams, the liquid will assemble almost exclusively along the lines of the skeleton of Plateau borders. The 'decoration' of this skeleton with liquid leaves the geometrical properties of the structure essentially unchanged, at least for sufficiently small liquid fractions. However, a closer look at the shape of the Plateau borders containing a non-vanishing amount of liquid shows that corrections are to be made. One has to consider a non-zero Laplace pressure of the liquid respective to the surrounding gas compartments. Consequences of the decoration of the foam skeleton with liquid have been discussed by Kern and Weaire [11]. The Laplace under-pressure in the Plateau border is responsible for a negative energy contribution related to the liquid content in the film edges. This can be described mathematically by a negative line tension of the film edges. Such a line tension may change, for example, the equilibrium angles of three films when the connecting edge is not straight. Géminard et al. [12] have demonstrated this in a particularly simple soap film structure. They have chosen the geometry of catenoid soap films with a central film spanning in the waist of the catenoids. They found a monotonous relation between the contact angle and the liquid content in the Plateau border. The angle between the two catenoid films increases with the liquid content, i.e. the central planar film is slightly larger than expected for a dry foam. The Plateau border is consequently longer than for an ideal dry film. Besson et al. [13] have confirmed this observation and performed dynamical measurements of the Plateau angle in soap films under mechanical strain. Fortes and Teixeira have calculated Plateau borders in soap foams for a number of different cell geometries, including cells in $3 \mathrm{D}$ foams $[14,15]$ and hemispherical bubbles on solid substrates [15-18]. They also obtained deviations of the Plateau border shape from a $120^{\circ}$ angle in $3 \mathrm{D}$ foam cell arrangements [14].

Besides aqueous solutions, which have been extensively studied in the past, foams of other complex fluids have been a topic of interest in recent research. Foams can be formed, e.g., by lamellar ionic liquid crystals [19-21] and by calamitic smectics [22]. Smectic films behave in many respects similar to soap films, although their internal structure is in general quite different. Ageing and structural properties of smectic foams have been reported [22]. In the essential characteristics, smectic foams behave like aqueous foams. It has been shown earlier that smectic phases form catenoids, too. These structures have been used, e.g. for studies of oscillations [23] and collapse dynamics [24]. Smectic catenoids can be prepared with well defined film thicknesses (Fig. 1), and uniform smectic catenoids are not susceptible to drainage. In this communication, we report the analysis of Plateau border geometries of smectic films in equilibrium.

\section{Experiment}

We explore the same geometry as used by Géminard et al. [12] in the previous study of soap film catenoids. The structure under investigation consists of a flat circular smectic liquid crystal (LC) film spanned between two catenoid smectic films 


\section{Page 3 of 10 \\ Philosophical Magazine \& Philosophical Magazine Letters}

1

2

3

4

5

6

7

8

9

10

11

12

13

14

15

16

17

18

19

20

21

22

23

24

25

26

27

28

29

30

31

32

33

34

35

36

37

38

39

40

41

42

43

44

45

46

47

48

49

50

51

52

53

54

55

56

57

58

59

60

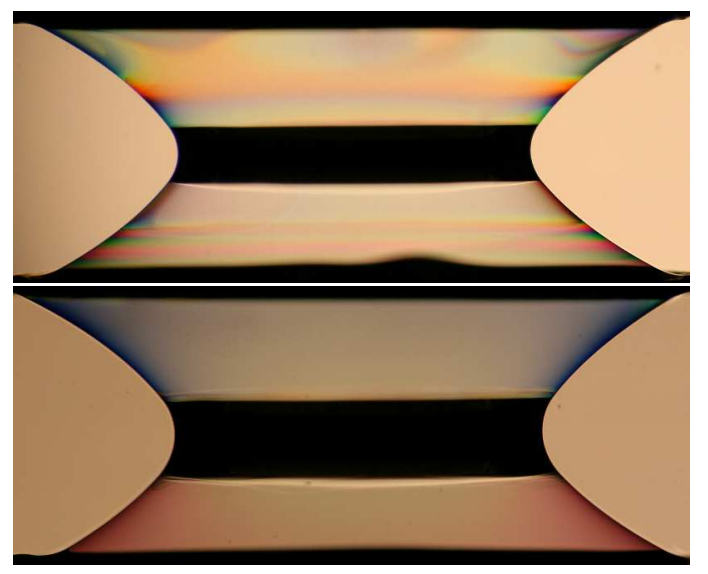

Figure 1. Images of catenoid structures with non-uniform film thickness before equilibration (top) and final uniform film thickness (bottom). The catenoids with the uniform film thickness are less than 50 layers thick, the non-uniform catenoids have thicknesses of several hundred layers.

(Fig. 2a). The setup consists of two vertically moveable coaxial tubular supports (diameter $D=10 \mathrm{~mm}$ ) with beveled ends. The films are observed with a digital camera, see Fig. 3a. The LC material used is the standard substance 4-octyl-4'cyanobiphenyl (8CB) (commercially produced by Synthon $\mathrm{GmbH}$ ) with a purity of $99.6 \%$ (GC). The chemical structure and the mesomorphism are sketched in Fig. 3b. All experiments were performed at room temperature $\left(25^{\circ} \mathrm{C}\right)$ in the smectic A mesophase.

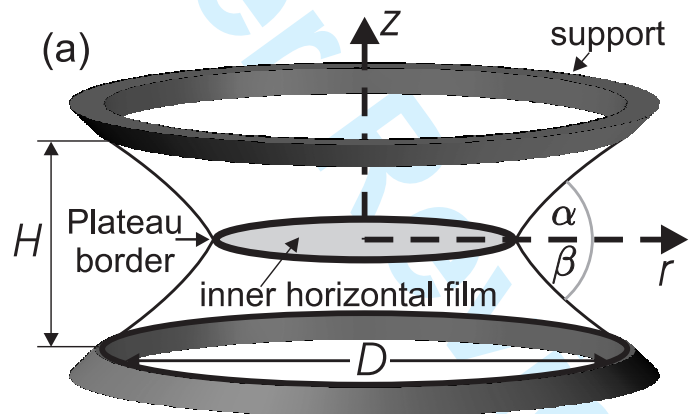

(b)

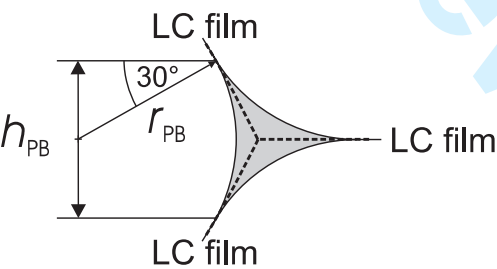

Figure 2. (a) Sketch of the three-film geometry. A circular LC-film (light grey) surrounded by a Plateau border is suspended between two catenoidal LC-films.(b) Profile of the Plateau border in the central cross section. Simple geometric relations show that when the Plateau border meets the adjacent film tangentially,

$h_{\mathrm{PB}}$ can be set equal to $r_{\mathrm{PB}}$.

Plateau borders of freely suspended smectic films are prepared in a three-step procedure. First, we apply a small amount LC-material onto the lower support tube. Second, we contact the coaxial tubes so that the liquid crystal completely connects the two tube ends. Then we pull a catenoid LC film between the two supports. The distance between the supports is increased beyond the critical distance for catenoid rupture $\left(H_{\mathrm{c}} \approx 0.66274 D\right)$. When the catenoid collapses, thin circular films remain on each tube end. One of them is kept. We bring the tubes in contact again, and then pull another catenoid film. Now it contains a central film that spans the waist of the outer film. The resulting two catenoids and the central film 
(a)

digital
camera $\begin{gathered}\text { macro } \\ \text { lens }\end{gathered}$

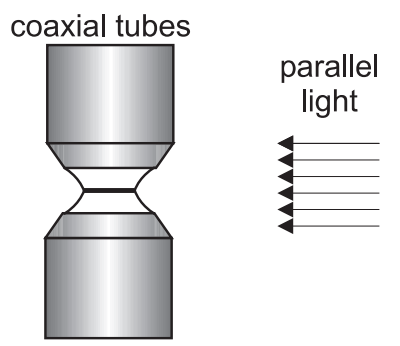

(b)

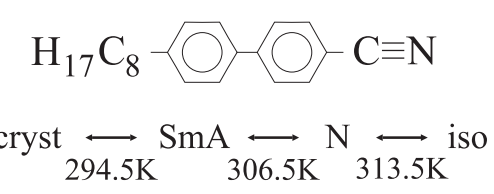

Figure 3. (a) Sketch of the experimental setup. The three-film geometry is prepared between two coaxial cylindrical tubes. Tube diameters are $10 \mathrm{~mm}$; (b) chemical structure and mesophases of the liquid crystal $8 \mathrm{CB}$.

are stable as long as the distance between the two supports remains smaller than $\approx 0.405 D$. When the two tube ends are separated further, the films collapse again. Thus, all experiments are performed at separations $H<0.4 D$. The LC content in the Plateau border is more or less affected by the initial amount of liquid crystal at the tube ends, it can be controlled to a certain extend. More material leads to thicker Plateau borders.

The catenoids are illuminated with monochromatic parallel light. This facilitates the analysis of the pictures. Photos are taken with a Canon EOS 400D digital camera with macro lens. Figure 4 shows a typical side view of the experimental situation. The films, with thicknesses between $100 \mathrm{~nm}$ and a few micrometers, are transparent. They show characteristic interference colors when illuminated with white light, or interference stripes under monochromatic illumination, from which the local film thickness can be determined. In this work, we did not particularly care to achieve uniformly thick films. The Plateau borders appear as dark bands. Here, the incident light is deflected because entrance and exit surfaces of light beams are not parallel to each other inside the Plateau border region. The edges of the Plateau borders appear very sharp in the transmission images, so that $h_{\mathrm{PB}}$ can be determined with an accuracy of about $20 \mu \mathrm{m}$.

We note that in soap films, one can show by simple geometric relations that the Plateau border radius $r_{\mathrm{PB}}$ can be set equal to the visible high $h_{\mathrm{PB}}$. This is sketched in Fig. 2b. For reasons detailed in the discussion, we will not use this equality $a$ priori but relate all Plateau border size measurements to the visible height $h_{\mathrm{PB}}$.

Image analysis is performed with MATLAB software. It consists of the following steps: the picture is converted into grayscale and cropped, background is subtracted, the catenoid profile is extracted with an edge detection routine. From the extracted shape of the catenoid, we define the rotational axis $z$ and the coordinates $r(z)$ (compensating potential slight deviations of the camera axis from the catenoid axis). Then, the catenoid profiles below and above the central Plateau border are fitted to hyperbolic cosine functions.

$$
r(z)=a \cosh \frac{\left(z-z_{0}\right)}{a}
$$

Technically, we perform a coordinate transformation $z \rightarrow z^{\prime}$ so that $z^{\prime}=0$ at the support, and $r\left(z^{\prime}\right)=a \cosh \left(\operatorname{arcosh}\left(R_{0} / a\right)-z^{\prime} / a\right)$, so that only one free parameter, $a$, remains. $R_{0}=z^{\prime}(0)=D / 2$ is the radius of the catenoid film at the support tube. 
The result of image processing is shown in Fig. 4. The constant $z_{0}$ would correspond to the position of the waist (minimal catenoid radius) in absence of the central film, the parameter $a$ guarantees that the boundary conditions are matched. Ideally, the central film is in a mirror plane of the structure, but due to minimal differences in the positions of the catenoid films at the supports, there may be slight differences between the upper and lower catenoids, leading to minor differences between the angles $\alpha$ and $\beta$. Also, gravitational effects influence the upper (green line in Fig. 4) and lower (red line) catenoid films. These deviations do not affect the experimental results, they cancel out in our evaluation procedure.

As a final step, we calculate the point of intersection of the two hyperbolic cosine functions. The inner film radius $R$ is determined as the distance between the point of intersection and the axis of rotation. The angles $\alpha$ and $\beta$ are evaluated from the slopes of the fitted catenary functions in the point of intersection. The angle $\theta$ is the sum of $\alpha$ and $\beta$. This procedure agrees with the evaluation scheme in refs. $[12,13]$. We should mention at this point that $\alpha$ and $\beta$ are not equivalent to the angles of the films at the outer edges of the Plateau border. Because of the curvature of the fit functions, the latter angles are systematically smaller than $60^{\circ}$ for both catenoids, in particular when the Plateau border is very broad. They are irrelevant for the interpretation of our experiments. The decoration lemma for Plateau borders [25] provides the justification of our data analysis. Weaire has pointed out that the prediction of $120^{\circ}$ angles no longer holds at the Plateau border edges of curved films [26, 27].
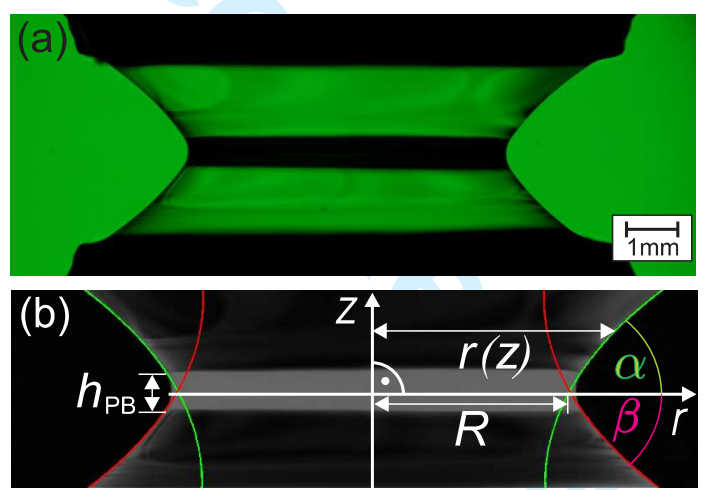

Figure 4. (a) Image of the experimental situation taken with the digital camera. The circular inner LCfilm is masked by the thick Plateau border. (b) Picture after image processing and edge detection. The red and green lines show the fitted hyperbolic cosines.

\section{Results}

First, it has to be verified that the films are in equilibrium when the images are recorded. Besson et al. [13] found relaxation times of the order of $10 \mathrm{~s}$ for their soap films. In smectic material, one may expect even longer equilibration phases, because of the higher viscosities of smectics. In order to test the equilibration time, we have recorded images of films with different Plateau border thicknesses (thick border, ratio $h_{\mathrm{PB}} / R=0.20$, and thin border, ratio $h_{\mathrm{PB}} / R=0.04$ ) in intervals of 1 min (Fig. 5). In both geometries, the structures have reached constant equilibrium angles $\theta$ in less than 5 minutes. It was not intended here to determine the equilibration dynamics, but the measurement certifies that angles determined after 10 minutes can be safely regarded as equilibrium values.

In a second set of experiments, we search for hysteresis effects. We prepare the films and change the distance $H$ of the supports stepwise. In order to avoid collapse 


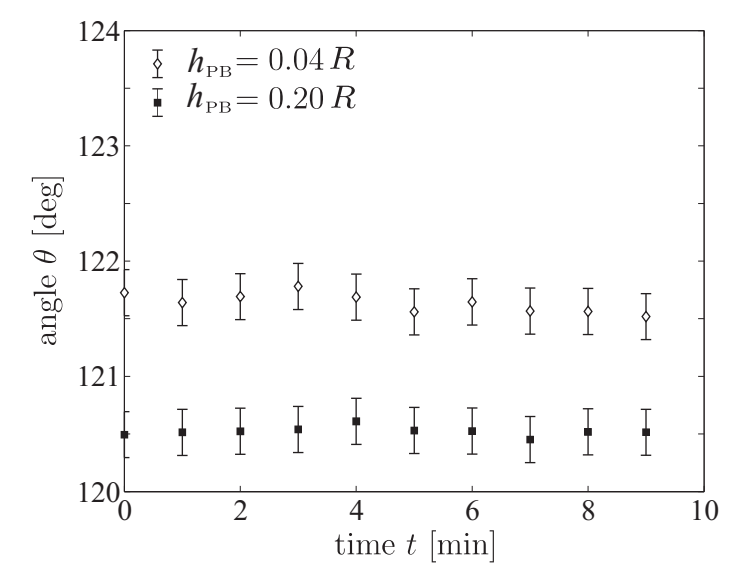

Figure 5. Time dependence of the angle $\theta=\alpha+\beta$ of the two catenoid films after the distance between the two support tubes has been adjusted.

of the catenoids we pull the structure first to $H=3.5 \mathrm{~mm}$, then we reduce the support distances stepwise to $H=2.5 \mathrm{~mm}$. Afterwards, we increase the distance again to $H=3.7 \mathrm{~mm}$. The results are shown in Fig. 6 . We do not find any hysteresis effects within the experimental accuracy. The slight decrease of $\theta$ with decreasing tube distance $H$ is the consequence of a slightly varying ratio $h_{\mathrm{PB}} / R$. With decreasing tube separation $H$, both the radius of the central film and the Plateau border length increase. When the liquid content in the border remains approximately constant, $h_{\mathrm{PB}}$ consequently shrinks. This is shown in Fig. 7 .

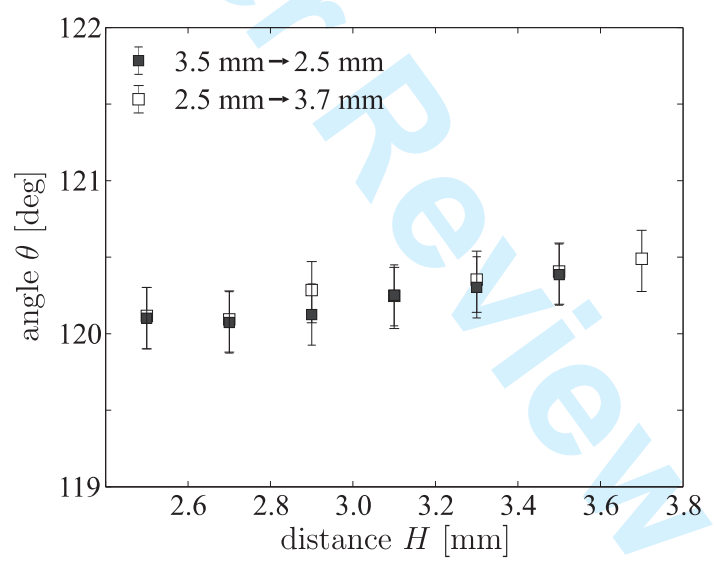

Figure 6. The variation of the angle $\theta$ during the stepwise variation of the support distance $H$. Within experimental accuracy, angles measured during increment of $H$ (open symbols) and angles measured during decrement of $H$ (solid symbols) coincide.

Finally, we have measured the dependence of the angle $\theta$ on the ratio $h_{\mathrm{PB}} / R$. These data have been obtained from a large number of three-film structures, they are shown in Fig. 8. There is a clear tendency for the increase of $\theta$ with increasing liquid content in the Plateau borders. In linear approximation, the experimental data can be described by the relation

$$
\theta\left(\frac{h_{\mathrm{PB}}}{R}\right)=6.84^{\circ} \cdot \frac{h_{\mathrm{PB}}}{R}+120^{\circ} .
$$

For $h_{\mathrm{PB}} / R \rightarrow 0$ ('dry' limit, vanishing liquid content) we regain the theoretical value for dry foam $\theta=120^{\circ}$. The dashed line sketches the result obtained for a soap film by Géminard et al. [12], when one sets $r_{\mathrm{PB}}=h_{\mathrm{PB}}$. 


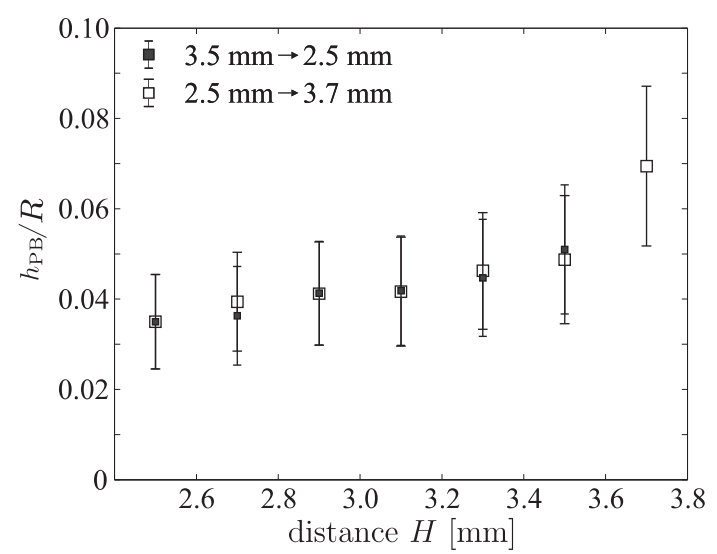

Figure 7. Variation of the Plateau border width $h_{\mathrm{PB}}$ (in units of the radius of the inner film $R$ ) during stepwise variation of the tube distance $H$. Symbols as in Fig. 6.

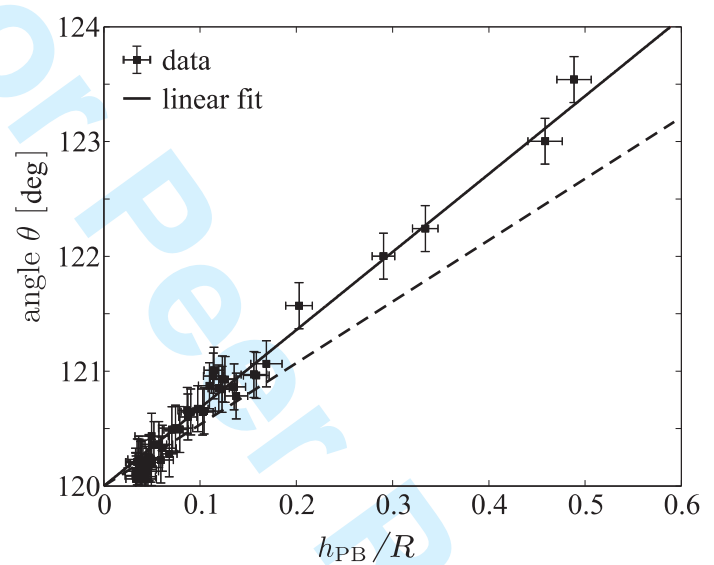

Figure 8. The angle $\theta$ is increasing with increasing ratio $h_{\mathrm{PB}} / R$. The dependence $\theta\left(h_{\mathrm{PB}} / R\right)$ can be described with a linear relation. The prediction of the model, Eq. (4), is shown as a dashed line.

\section{Discussion and Summary}

Our measurements of the angles of Plateau borders in smectic A films confirm qualitatively the soap film results by Géminard et al. [12]. The angles between the two outer catenoid films are larger than $120^{\circ}$. Their angle $\theta$ depends linear on the ratio $r_{\mathrm{PB}} / R$. Thicker Plateau borders lead to larger values of $\theta$. There is no qualitative difference between smectic LC films and soap films. The 'decoration energy' is of the same order of magnitude as in soap films. Quantitatively, however, the slope in the linear relation, Eq. (2), in the smectic films is somewhat steeper than in soap films. In order to discuss this point, we make a distinction between our measured quantity, $h_{\mathrm{PB}}$, and the Plateau border radius $r_{\mathrm{PB}}$.

Using the concept of the negative line tension, one may estimate the dependence of $\theta\left(r_{\mathrm{PB}} / R\right)$ from a model assumption of the Plateau border shape [12]. The line tension for a Plateau border cross section formed by three circular arcs (Fig. 2) is $T=-(\sqrt{3}-\pi / 2) \gamma r_{\mathrm{PB}}$, where $\gamma$ is the surface tension of the LC. Then, the force balance in the central film plane, along the radial direction, requires

$$
\cos \alpha+\cos \beta=1+\frac{T}{2 \gamma R}=1-\frac{(\sqrt{3}-\pi / 2)}{2} \frac{r_{\mathrm{PB}}}{R}
$$

Since $\alpha$ and $\beta$ are close to $60^{\circ}$, one can expand the two angles linearly in powers of the small deviations $\alpha^{\prime}=\alpha-60^{\circ}, \beta^{\prime}=\beta-60^{\circ}: \cos \alpha \approx 1 / 2-\sqrt{3} \alpha^{\prime} / 2$ and 
$\cos \beta \approx 1 / 2-\sqrt{3} \beta^{\prime} / 2$, so that

$$
\alpha^{\prime}+\beta^{\prime}=\theta-120^{\circ} \approx\left(1-\frac{\pi}{\sqrt{12}}\right) \frac{r_{\mathrm{PB}}}{R}
$$

This model would predict a slope of $5.33^{\circ} \cdot r_{\mathrm{PB}} / R$, which is in excellent agreement with the soap film measurements [12], but $25 \%$ below the value measured for the smectic films. Even though the measured angles for narrow Plateau borders agree with the model within the experimental uncertainty, there is a clear and systematic deviations for broad Plateau borders. Optical aberrations or similar systematic errors can be ruled out, since the angle for dry foam is accurately reproduced in films with low liquid content in the Plateau border $\left(h_{\mathrm{PB}} \rightarrow 0\right)$.

It is not immediately obvious why we find a slightly higher negative line tension in the smectic systems. Therefore it may be appropriate to discuss differences to soap films. Whereas the outer geometrical shapes of dry soap and smectic foams are quite similar, the internal structures differ because of the smectic layering. Particularly in the Plateau borders, smectic layers form dislocations, with a positive contribution to the free energy density (Fig. 9). Complex layer distortions, for example disordered domains or focal conics, may be found in the meniscus. Optically, we are not able to extract the inner structure of thick menisci. It is
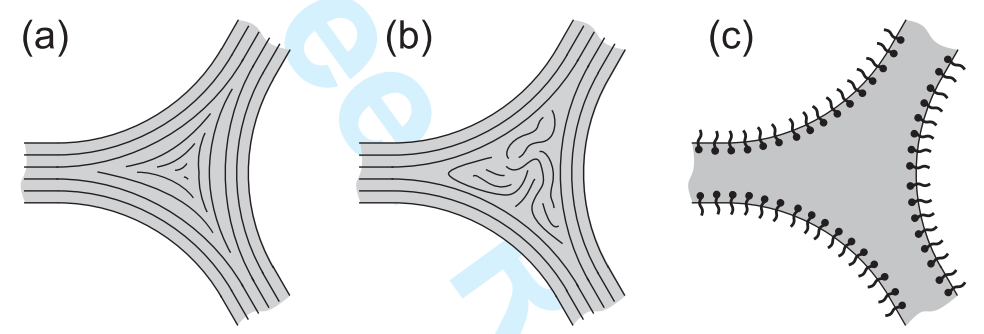

Figure 9. Sketch of the internal structure of the Plateau borders for smectic films (a,b) and soap films (c). The smectic film will develop dislocation lines, like in the meniscus of freely suspended films [28], as shown in (a). In thick Plateau borders, disordered multidomains may be found (b).

reasonable to assume that the disorder of the smectic layers gives a positive contribution to the decoration energy. This would lower the expected deviations of the angle $\theta$ from $120^{\circ}$. On the other hand, these elastic contributions are negligibly small with respect to the surface tension effects. An order of magnitude estimation may demonstrate this: The free energy density in elastic distortions of the director, irrespective of their detailed shapes, is of the order of $K q^{2}$ where $K$ is an effective elastic constant (of the order of $10 \mathrm{pN}$ ) and $q$ is the wave number of deformations, roughly of the order of $1 \mu \mathrm{m}^{-1}$. The cross section of the Plateau border is of the order of $0.1 r_{\mathrm{PB}}^{2}$, this gives an energy per length of $1 \mathrm{Nm}^{-2} \cdot r_{\mathrm{PB}}^{2}$. On the other hand, the surface energy per length of the border is of the order of $\pi \gamma r_{\mathrm{PB}} \approx 0.1$ $\mathrm{Nm}^{-1} \cdot r_{\mathrm{PB}}$. If one assumes that the Plateau border radius is $100 \mu \mathrm{m}$, the two quantities relate to each other as $10^{-8} \mathrm{~N}: 10^{-5} \mathrm{~N}$. Even if we take into account that defect structures are also present in the smectic meniscus, with energy densities roughly comparable to the elastic distortions, the surface energy contributions are larger than these bulk terms by more than two orders of magnitude.

Another possible explanation could be the assumption of a non-zero contact angle $\varphi$ between the Plateau border and the adjacent films (see Fig. 10), as opposed to soap films where the circular arcs meet the films tangentially. Such finite contact angles between meniscus and film have been measured quantitatively, e.g. [29, 30]. This would result in a more effective surface reduction of the Plateau border by LC decoration, and consequently to an even lower effective line tension. The model 


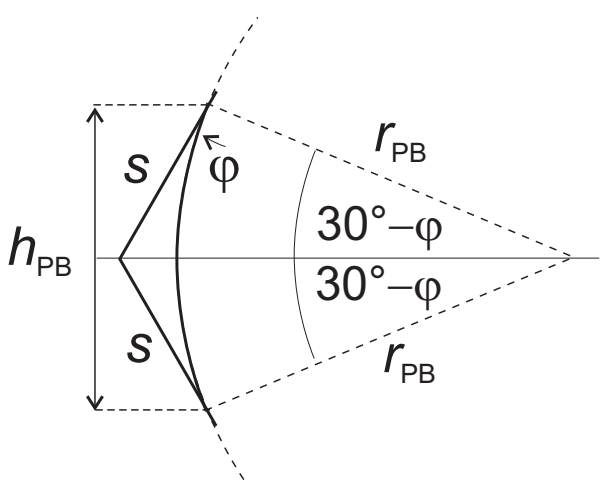

Figure 10. Left: Sketch of the different Plateau border profiles in the soap film model (surfaces meet tangentially, grey shaded area, dashed line) and the smectic film model (non-zero contact angle, solid lines). Right: geometrical quantities in the case of a non-zero contact angle between film and meniscus.

sketched on the right hand side of Fig. 10 shows that in the smectic film the visible height of the Plateau border is no longer equal to the Plateau border radius. Instead,

$$
h_{\mathrm{PB}}=2 r_{\mathrm{PB}} \sin \left(30^{\circ}-\varphi\right)
$$

so that the difference between the surface of the dry and the decorated Plateau borders becomes

$$
T=-\left(\sqrt{3}-\frac{\pi-6 \varphi}{4 \sin \left(30^{\circ}-\varphi\right)}\right) \gamma h_{\mathrm{PB}}
$$

For $\varphi=0$, this equation is identical with the value given for soap films. If we make a linearized small angle approximation for $\varphi$, we obtain

$$
T \approx-\left(\sqrt{3}-\frac{\pi-6 \varphi+\sqrt{3} \pi \varphi}{2}\right) \gamma h_{\mathrm{PB}} .
$$

For the angle between the two catenoids, one finds

$$
\theta=120^{\circ}+5.33^{\circ} \cdot r_{\mathrm{PB}} / R+0.161 \varphi \cdot r_{\mathrm{PB}} / R
$$

Qualitatively, this contribution provides the correct trend. But quantitatively, the result is disappointing. A back of the envelope calculation indicates that angles $\varphi$ of the order of $\approx 10^{\circ}$ are necessary to increase the slope of the theoretical (dashed) curve in Fig. 8 by $25 \%$. We have no quantitative data for the contact angle in the present material, but this angle would be about one order of magnitude larger than available literature data $[29,30]$ in smectic A. Moreover, such a large contact angle would be detectable in the catenoid profiles. In smectic C films, contact angles of the order of $\approx 5^{\circ}$ have been measured [31], and the influence of such contact angles on line tensions of Plateau borders should in principle be detectable, when smectic $\mathrm{A}$ and $\mathrm{C}$ films are compared.

Summarizing, the study of smectic films confirms the existence of a negative line tension in Plateau borders. It increases in good approximation linearly with the Plateau border height. The quantitative results for smectics are of the same order of magnitude as for soap films and as predicted by model [12]. Nevertheless, the reason for slight quantitative differences remains unclear. The reduced surface in a wet Plateau border with respect to the dry limit alone cannot explain the smectic 
A results. Defect and distortion energies in the smectic Plateau borders are too small to contribute to the line tension. Moreover, their contribution is opposite to the observed effect.

\section{Acknowledgments}

The authors acknowledge Marvin Weyland for participating in some of the experiments and DFG for financial support within Project STA 425/20. R. A. acknowledges support by the DAAD. We thank D. Weaire for useful comments.

\section{References}

[1] J.A.F. Plateau, Statique Expérimentale et Théorique des Liquides Soumis aux Seules Forces Moléculaires, Gauthier-Villars, Paris, 1873.

[2] F.J. Almgren and J.E. Taylor, Scientific American 235 (1976) p. 82-93.

[3] D. Weaire, The physics of foams, Clarendon Press, Oxford, 1999.

[4] D. Weaire, M.F. Vaz, P.I.C. Teixeira and M.A. Fortes, Soft Matter 3 (2007) p. 4757.

[5] A. Saint-Jalmes and D. Langevin, J. Phys.: Cond. Mat. 14 (2002) p. 9397-9412.

[6] A. Saint-Jalmes, Y. Zhang and D. Langevin, Eur. Phys. J. E 15 (2004) p. 53-60.

[7] P. Rognon, F. Molino and C. Gay, Europhys. Lett. 27 (2010) p. 253-260.

[8] P. Rognon and C. Gay, Eur. Phys. J. E 30 (2010) p. 291-301.

[9] M. Durand and H.A. Stone, Phys. Rev. Lett. 97 (2006).

[10] A.L. Biance, S. Cohen-Addad and R. Höhler, Soft Matter 5 (2009) p. 4672-4679.

[11] N. Kern and D. Weaire, Phil. Mag. 83 (2003) p. 2973-2987.

12] J.C. Géminard, A. Zywocinski, F. Caillier and P. Oswald, Phil. Mag. Lett. 84 (2004) p. 199-204.

13] S. Besson and G. Debrégeas, Eur. Phys. J. E 24 (2007) p. 109-117.

[14] M.A. Fortes and P.I.C. Teixeira, Phys. Rev. E 71 (2005) p. 051404.

$15]$ M.A. Fortes, P.I.C. Teixeira and A.M. Deus, J. Phys.: Cond. Matter 19 (2007) p. 246106.

[16] M.A. Fortes and P.I.C. Teixeira, Phil. Mag. Lett. 85 (2005) p. 2125.

[17] P.I.C. Teixeira and M.A. Fortes, Phys. Rev. E 75 (2007) p. 011404.

[18] P.I.C. Teixeira and M.A. Fortes, Colloids and Surfaces A 309 (2007) p. 3-6.

[19] M.H. Godinho, C. Cruz, P.I.C. Teixeira, A.J. Ferreira, C. Costa, P.S. Kulkarni and C.A.M. Afonso, Liq. Cryst. 35 (2008) p. 103.

[20] C. Cruz, M.H. Godinho, A.J. Ferreira, P.S. Kulkani, C.A.M. Afonso and P.I.C. Teixeira, Phil. Mag. Lett. 88 (2008) p. 741.

[21] A.J. Ferreira, C. Cruz, M.H. Godinho, P.S. Kulkani, C.A.M. Afonso and P.I.C. Teixeira, Liq. Cryst. 37 (2010) p. 377.

[22] T. Trittel, T. John and R. Stannarius, Langmuir 26 (2010) p. 7899-7904.

[23] M.B. Amar, P.P. Silvada, N. Limodin, A. Langlois, M. Brazovskaia, C. Even, I. Chikina and P. Pieranski, Eur. Phys. J. B 3 (1998) p. 197-202.

[24] F. Müller and R. Stannarius, Europhys. Lett. 76 (2006) p. 1102-1108.

[25] F. Bolton and D. Weaire, Phil. Mag. B: Physics of Condensed Matter; Electronic, Optical and Magnetic Properties 63 (1991) p. 795-809.

[26] J. Stavans and J.A. Glazier, Phys. Rev. Lett. 62 (1989) p. 1318-1321.

[27] D. Weaire, Phys. Rev. Lett. 64 (1990) p. 3202.

[28] P. Oswald and P. Pieranski, Smectic and Columnar Liquid Crystals: Concepts and Physical Properties Illustrated by Experiments, Taylor \& Francis, Boca Raton, 2005.

[29] F. Picano, P. Oswald and E. Kats, Phys. Rev. E 63 (2001) p. 021705.

[30] A. Poniewierski, P. Oswald and R. Hołyst, Langmuir 18 (2002) p. 1511-1517.

[31] K. Harth and R. Stannarius, Eur. J. Phys. E 28 (2009) p. 265. 